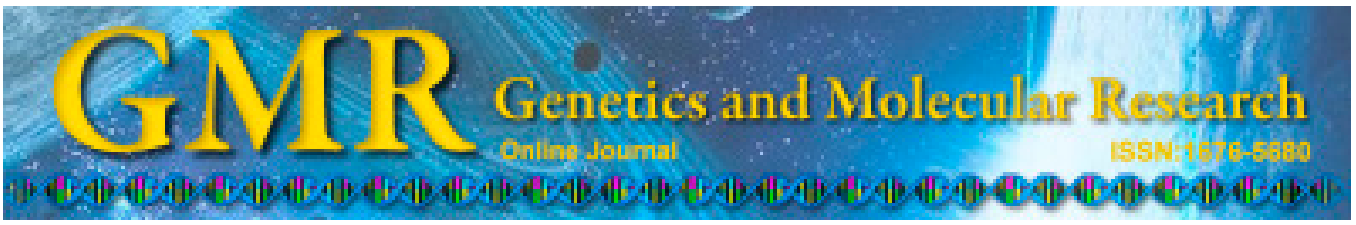

\title{
Identification of critical TF-miRNA-mRNA regulation loops for colorectal cancer metastasis
}

\author{
C. Wang ${ }^{1,2}$, D.Z. Hu ${ }^{1,2}$ and J.Z. Liu ${ }^{1,2}$ \\ ${ }^{1}$ Tianjin Medical University Cancer Institute and Hospital, \\ National Clinical Research Center for Cancer, Tianjin, China \\ ${ }^{2}$ Key Laboratory of Cancer Prevention and Therapy, Tianjin, China \\ Corresponding author: J.Z. Liu \\ E-mail: Do_JZLiu@126.com
}

Genet. Mol. Res. 14 (2): 5485-5495 (2015)

Received November 17, 2014

Accepted March 23, 2015

Published May 22, 2015

DOI http://dx.doi.org/10.4238/2015.May.22.19

\begin{abstract}
To explore the potential cause of colorectal cancer metastasis, gene expression profiles, GSE21510, and miRNA expression profiles, GSE48074, were downloaded from the Gene Expression Omnibus database. Differentially expressed genes in metastatic colorectal and non metastatic colorectal cancer compared with the normal samples were identified via the limma package in $\mathrm{R}$. The differentially expressed miRNAs in colorectal cancer samples with lymph node metastasis compared with those without lymph node metastasis were screened out by the some method. Differentially expressed genes that were upregulated in colorectal cancer samples with distant metastasis in comparison to that in samples without distant metastasis and normal samples were considered to play important roles in colorectal cancer metastasis. Functional enrichment analysis of these genes was conducted using the Database for Annotation, Visualization, and Integrated Discovery v6.7. Biological processes related to cell differentiation and cell proliferation were significantly enriched. $\mathrm{TF}$ (transcription factor)-miRNA-mRNA regulation loops were constructed by using the starBase and ChIPBase databases. Finally, six critical regulation loops were screened out. They were composed of two
\end{abstract}


TFs, two miRNAs, and three mRNAs. Some of these TFs, mRNAs, or miRNAs have previously been identified as critical targets in colorectal cancer metastasis. Additionally, several new targets were identified in our study, which may be helpful to improve metastatic colorectal cancer treatment.

Key words: ChIPBase; Colorectal cancer; DAVID; TF-miRNA-mRNA; Gene Expression Omnibus; StarBase

\section{INTRODUCTION}

Colorectal cancer (CRC) is one of the most common malignancies with high morbidity and mortality (Coghlin and Murray, 2015). In some developed Chinese cities such as Shanghai and Hong Kong, it has become the second most common cancer ( $\mathrm{Li}$ and $\mathrm{Ma}$, 2014). Metastasis is the major cause of death related to CRC (Zhou et al., 2014) and the most common distant metastasis site is the liver (Lupinacci et al., 2014). Some treatments for CRC with or without metastasis have been developed and oxaliplatin-based regimens are the most commonly used treatments to improve survival (Morris-Stiff et al., 2014). However, tumor metastasis is a complex process and its mechanisms remain ambiguous. Herszenyi et al. (2014) proved that proteolytic enzymes play an important role in CRC metastasis. Yesudhas et al. (2014) reported that TLR-4 was a critical target in CRC metastasis. In this study, we focused on exploring the potential cause of CRC metastasis and on screening molecular targets by the analysis of gene and miRNA expression profiles from microarray data.

miRNA is a small non-coding RNA with a length of 20-25 nucleotides. It is recognized as an important factor in gene regulation (Joshi et al., 2014; Zhu et al., 2014). miRNAs can target a gene and induce the repression of translation or post-transcriptional RNA degradation (Hu and Tang, 2014; Zhang and Wang, 2014). The deregulation of some miRNAs has been proven to be related to some diseases, including cancer. Shiah et al. (2014) proved that $m i R-329$ and $m i R-410$ were associated with the pathogenesis of oral squamous carcinoma through the analysis of miRNA expression profiles. A transcription factor (TF) is a protein that controls the transcription process through binding to specific sequences in a gene regulatory region (Latchman, 1993). TF can not only directly regulate the expression of mRNAs, but also that of miRNAs. Thus, TF deregulation may result in the deregulation of miRNAs or mRNAs and induce the appearance of some physical abnormalities.

RNA microarray, a method developed more than 20 years ago, can be used to quantitatively measure gene expression in a very high throughput manner (Debouck and Goodfellow, 1999). It becomes possible to explore the mechanisms or signatures for diseases at the gene level using RNA microarray. Using RNA microarrays, Xu et al. (2013) obtained a 18-gene signature for CRC through the analysis of differentially expressed genes (DEGs) in 216 patients with CRC than that in 181 controls. Clifford et al. (2010) found two single nucleotide polymorphisms that were significantly different between liver cancer samples and normal samples. The large amount of expression profiles generated from RNA microarray are stored in the Gene Expression Omnibus (GEO) database (http://www.ncbi.nlm.nih.gov/geo/), in which the data can be freely interpreted (Barrett et al., 2011). 
The starBase (http://starbase.sysu.edu.cn/) is a database that deciphers the regulation relationships between RNA and RNA, or protein and RNA by managing the 108 CLIP-Seq datasets from 37 independent studies (Li et al., 2014). It can provide miRNA-mRNA regulation pairs when using a different parameter setting such as the number of CLIP-Seq experiments that can support the pair and the number of software that predict the pair. The ChIPBase (http://deepbase.sysu.edu.cn/chipbase/) is an integrated resource and platform to decode TF binding sites, expression profiles, and transcriptional regulation of non-coding RNAs such as IncRNA, miRNA, and lincRNA via the interpretation of data from 543 CHIP-Seq experiments (Yang et al., 2013). The ChIPBase can provide TF-miRNA regulation pairs when using different parameter settings such as the regulatory regions.

In this study, through the analysis of CRC mRNA and miRNA expression profiles from the GEO database, we investigated the potential cause of CRC metastasis. As a result, six TF-miRNA-mRNA regulation loops that may play critical roles were identified. The TFs, miRNAs, and mRNAs in those loops may serve as important targets for the treatment of metastatic CRC. The workflow of this study is presented in Figure 1.

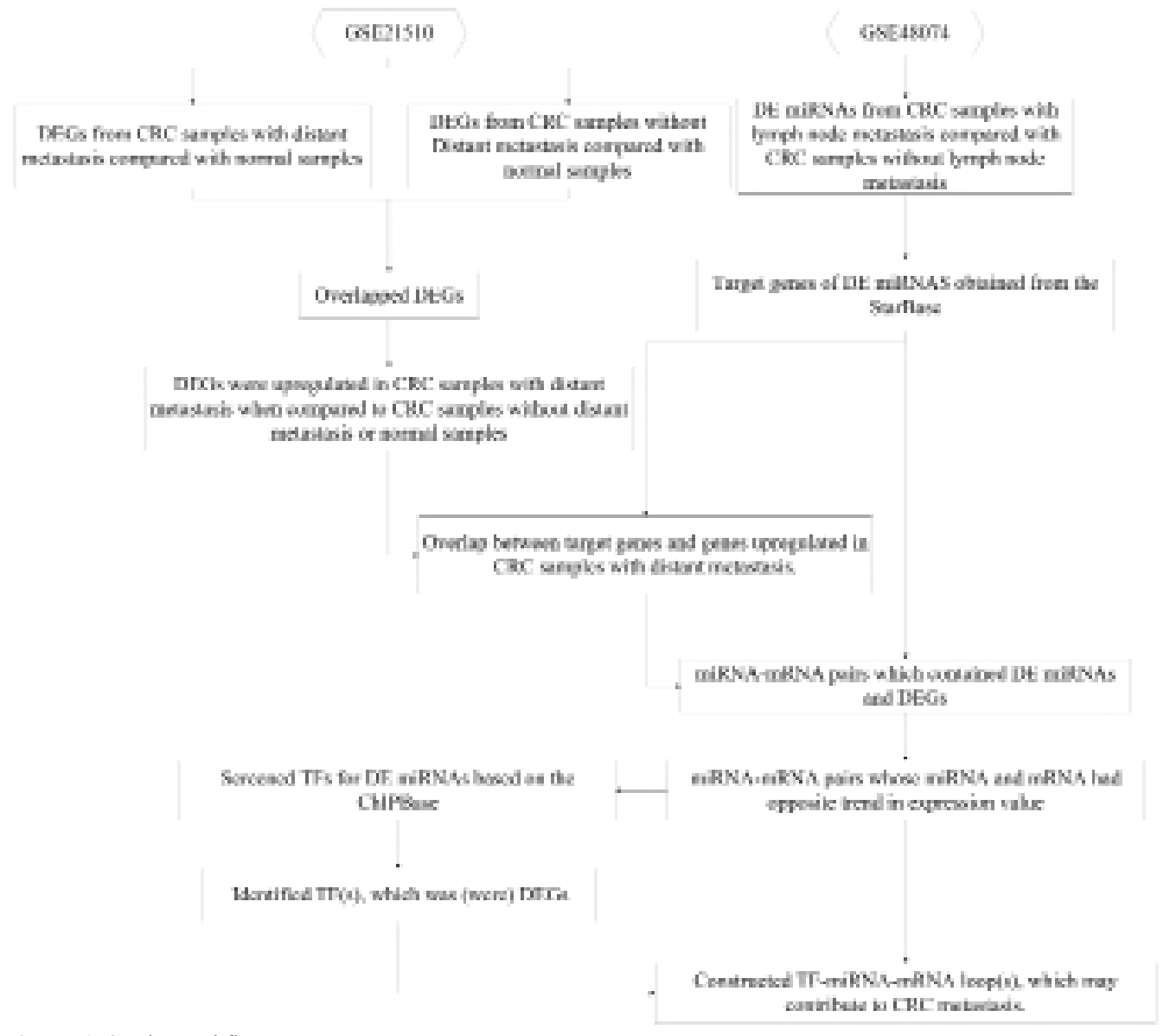

Figure 1. Study workflow. 


\section{MATERIAL AND METHODS}

\section{Microarray data}

The mRNA expression profiles were downloaded from the GEO database using the accession No. GSE21510 (Tsukamoto et al., 2011), which contains a total of 148 samples, including 18 normal colorectal tissue samples, 76 CRC samples without distant metastasis, and 54 CRC samples with distant metastasis. The GSE21510 dataset is based on GPL570 [HG-U133_Plus_2] Affymetrix Human Genome U133 Plus 2.0 Array. The miRNA expression profiles (GSE48074) based on GPL11487 Aglient-021827 Human miRNA Microarray [miRNA_107_Sep09_2_105] were also downloaded from the GEO database. Briefly, it is composed of four CRC samples with lymph node metastasis and four CRC samples without lymph node metastasis.

\section{Preprocessing of the microarray data}

The raw data in CEL format were downloaded and preprocessed by using R (Huber et al., 2015). Briefly, the expression values were $\log 2$-tansformed and standardized by using the robust multi-array average (RMA) method. The probe IDs were then changed into gene symbols by using annotation packages for the mRNA and miRNA microarray platforms. Finally, we summarized the expression levels of the probe sets according to gene symbols.

\section{Identification of DEGs/differentially expressed (DE) miRNAs}

The limma (Diboun et al., 2006) package in $\mathrm{R}$ was used to identify DEGs and DE miRNAs. Genes and miRNAs with $\mathrm{P}<0.05$ and $\mid \log 2$ (fold change) $\mid>1$ were kept. Here, two groups of DEGs (DEGs from CRC samples with/without distant metastasis than those of normal samples) and one group of DE miRNAs (DE miRNAs from CRC samples with lymph node metastasis than those of CRC samples without lymph node metastasis) were screened out.

\section{Identification of critical DEGs for CRC metastasis}

Gaiteri et al. (2014) indicated that, if the average expression level of a gene module was higher in patient samples than in normal samples, the genes in this module are more likely to be related to the cause of the disease. To identify the critical DEGs involved in CRC metastasis, we detected DEGs that were upregulated in CRC samples with distant metastasis than those of CRC samples without distant metastasis and normal samples. These genes were referred to as DEG1. Functional enrichment analysis of DEG1 was conducted by using the Database for Annotation, Visualization, and Integrated Discovery (DAVID; http://david.abcc. ncifcrf.gov/) v6.7 and GO terms with $\mathrm{P}<0.05$ were screened out.

\section{Identification of critical TF-miRNA-mRNA regulation loops}

We obtained the miRNA-mRNA regulation pairs for the DE miRNAs based on the following parameter settings: Number of supported experiments $\geq 1$ (at least one CLIP-Seq 
experiment supported the miRNA-mRNA pair), number of cancer types $\geq 1$ (miRNA expression and its target mRNA were inversely correlated in at least one cancer type), and program number $\geq 1$ (at least one software predicted the miRNA-mRNA pair) in the starBase. We retained the miRNA-mRNA pairs if the mRNA belonged to the DEG1 group and miRNA expression level was inversely correlated to the target mRNA expression level in this study. For miRNAs in miRNA-mRNA pairs, we obtained the TF-miRNA regulation pairs based on the ChIPBase setting with upstream and downstream to 5 -kb regulatory regions. TF-miRNA pairs in which the TF belongs to the DEGs of CRC samples with or without distant metastasis and for which TF and miRNA expression levels were positively correlated were screened out. The critical TF-miRNA-mRNA regulation loops were obtained based on these TF-miRNA and miRNA-mRNA pairs.

\section{RESULTS}

\section{DEGs and DE miRNAs}

With a cutoff of $\mathrm{P}<0.05$ and $\mid \log 2$ (fold change) $\mid>1$, a total of 2851 DEGs from CRC samples without distant metastasis and 2029 DEGs from CRC samples with distant metastasis were identified when compared with normal samples. There were 1994 overlapped DEGs among the two DEG datasets. Moreover, $7 \mathrm{DE}$ miRNAs from CRC samples with lymph node metastasis were identified than those of CRC samples without lymph node metastasis. These include 5 downregulated and 2 upregulated miRNAs (Table 1).

Table 1. Comparison of differentially expressed miRNAs in CRC samples with lymph node metastasis and
CRC samples without lymph node metastasis.
\begin{tabular}{lcr}
\hline miRNA & Log FC & P value \\
\hline hsa-miR-152 & 1.248181 & 0.001254 \\
hsa-miR-551b & 1.431937 & 0.001514 \\
hsa-miR-19b & -1.1882 & 0.020905 \\
hsa-miR-29b & -1.32928 & 0.031561 \\
hsa-miR-99a & 1.202455 & 0.031916 \\
hsa-miR-141 & -1.42915 & 0.044461 \\
hsa-miR-194 & -1.01124 & 0.047281 \\
\hline
\end{tabular}

Log FC: $\log 2$ (fold change).

\section{Critical DEGs and functional enrichment analysis}

Among the 1994 overlapping genes, 49 genes were found to be upregulated in CRC samples with distant metastasis than in the ones without distant metastasis and normal samples (Table 2). The heatmap of the average expression level of these 49 genes in CRC samples with/without distant metastasis and normal samples are shown in Figure 2. Functional enrichment analysis indicated that these genes are mainly involved in the process of cell differentiation, cell proliferation, cell localization, and cell-cell adhesion. Erythrocyte differentiation and homeostasis were the top two most significant GO terms, which have been reported to be involved in CRC metastasis. Ochiai et al. (2014) indicated that erythropoiesis could increase serum iron, which is a convenient predictor of the response to chemotherapy in patients with advanced CRC. Vali et al. (2008) demonstrated that the ATP level in erythrocytes was associated with colorectal liver metastasis. The enriched GO terms are shown in Table 3. 
Table 2. Comparison of upregulated DEGs in CRC samples with distant metastasis and CRC samples without distant metastasis or normal samples.

\begin{tabular}{lcclcc}
\hline Gene & Log FC1 & Log FC2 & Gene & Log FC1 & Log FC2 \\
\hline AMIGO2 & 2.48 & 2.26 & LGR6 & 1.253915 & 1.068486 \\
BMP4 & 1.90 & 1.50 & LINC00888 & 1.202353 & 1.171287 \\
CLDN2 & 2.33 & 2.09 & LPL & 2.063578 & 1.299128 \\
CYP4F3 & 1.15 & 1.11 & LY6E & 1.238197 & 1.176091 \\
DACT1 & 1.57 & 1.08 & NMU & 1.622998 & 1.573041 \\
DEFA5 & 1.73 & 1.65 & OR51E1 & 1.655626 & 1.582115 \\
DEFA6 & 1.43 & 1.34 & PHLDA1 & 1.632322 & 1.610682 \\
DPYSL2 & 1.19 & 1.09 & PLAG1 & 1.619089 & 1.572133 \\
DSG3 & 1.29 & 1.22 & PLEKHG4 & 1.114769 & 1.023301 \\
EDAR & 1.55 & 1.31 & PRTFDC1 & 1.163461 & 1.100993 \\
EMG1 & 1.25 & 1.24 & PTPN13 & 1.058221 & 1.000699 \\
ETV4 & 1.14 & 1.02 & REG1A & 1.771995 & 1.131865 \\
FAN1 & 1.15 & 1.13 & REG1B & 1.730113 & 1.162919 \\
FAP & 2.01 & 1.94 & RNF182 & 1.365853 & 1.236283 \\
FLVCR1 & 1.11 & 1.01 & S100A2 & 1.464457 & 1.111458 \\
GEM & 1.26 & 1.14 & SKAP1 & 1.329612 & 1.101233 \\
GNG4 & 1.13 & 1.07 & STC16A4 & 1.864104 & 1.796496 \\
GRAMD1A & 1.09 & 1.06 & TCN1 1 & 1.497874 & 1.194649 \\
GSAP & 2.07 & 1.96 & TIMP1 & 2.32957 & 2.002145 \\
GSPT2 & 1.21 & 1.17 & TMEM71 & 1.358588 & 1.304934 \\
HS6ST2 & 2.44 & 2.32 & TMTC4 & 1.110418 & 1.396431 \\
IL33 & 1.91 & 1.88 & TRFRSF11B & 1.803088 & 1.080725 \\
KLK6 & 1.56 & 1.25 & & 1.054841 & 1.179123 \\
KRT6B & 1.50 & 1.14 & & \\
LEMD1 & 2.03 & 1.96 & & & \\
\hline
\end{tabular}

Log FC1: $\log 2$ (fold change) of CRC samples with distant metastasis than normal samples; $\log$ FC2: $\log 2$ (fold change) of CRC samples without distant metastasis than normal samples.

\section{Table 3. Enriched GO terms for the 49 critical genes.}

\begin{tabular}{llll}
\hline Category & Term & P value & Genes \\
\hline BP & Erythrocyte differentiation & 0.00613 & BMP4, FLVCR1, TIMP1 \\
BP & Erythrocyte homeostasis & 0.00790 & BMP4, FLVCR1, TIMP1 \\
BP & Growth & 0.01350 & BMP4, KLK6, LY6E, FLVCR1 \\
BP & Myeloid cell differentiation & 0.02664 & BMP4, FLVCR1, TIMP1 \\
BP & Regulation of cellular localization & 0.02992 & BMP4, STXBP1, EDAR, NMU \\
BP & Homeostasis of cell number & 0.03046 & BMP4, FLVCR1, TIMP1 \\
BP & Defense response to fungus & 0.03499 & DEFA6, DEFA5 \\
BP & Killing cells of another organism & 0.03764 & DEFA6, DEFA5 \\
BP & Cell-cell adhesion & 0.03920 & AMIGO2, DSG3, STXBP1, CLDN2 \\
\hline
\end{tabular}

Table note: BP: Biological Process.

\section{Critical TF-miRNA-mRNA loops}

Based on the starBase, a total of 1977 miRNA-mRNA regulation pairs for the $7 \mathrm{DE}$ miRNAs were obtained, of which four pairs contained mRNAs that belonged to DEG1, (hsamiR-29b)-PLAG1, (hsa-miR-29b)-LPL, (hsa-miR-141)-PLAG1, and (hsa-miR-141)-STXBP1. Moreover, miRNA expression levels were negatively correlated with the expression levels of its target mRNA in all four pairs. TFs that may regulate miRNAs identified in the four miRNA-mRNA pairs were obtained from the ChIPBase. Eighty seven TFs were obtained 
and three of them belonged to the DEGs from CRC samples with or without distant metastasis, E2F6, Fosl2, and TCF7L2. However, only Fosl2 and TCF7L2 expression levels were positively correlated with the expression levels of their target miRNAs and three TF-miRNA regulation pairs were obtained, Fosl2-(hsa-miR-141), TCF7L2-(hsa-miR-141), and TCF7L2(hsa-miR-29b). Based on the four miRNA-mRNA pairs and the three TF-miRNA pairs, a total of six TF-miRNA-mRNA regulation loops, Fosl2-(hsa-miR-141)-PLAG1, Fosl2-(hsamiR-141)-STXBP1, TCF7L2-(hsa-miR-141)-PLAG1, TCF7L2-(hsa-miR-141)-STXBP1, TCF7L2-(hsa-miR-29b)-PLAG1, and TCF7L2-(hsa-miR-29b)-LPL were obtained and thought to play important roles in CRC metastasis. The regulation network composed of these regulation loops is shown in Figure 3.
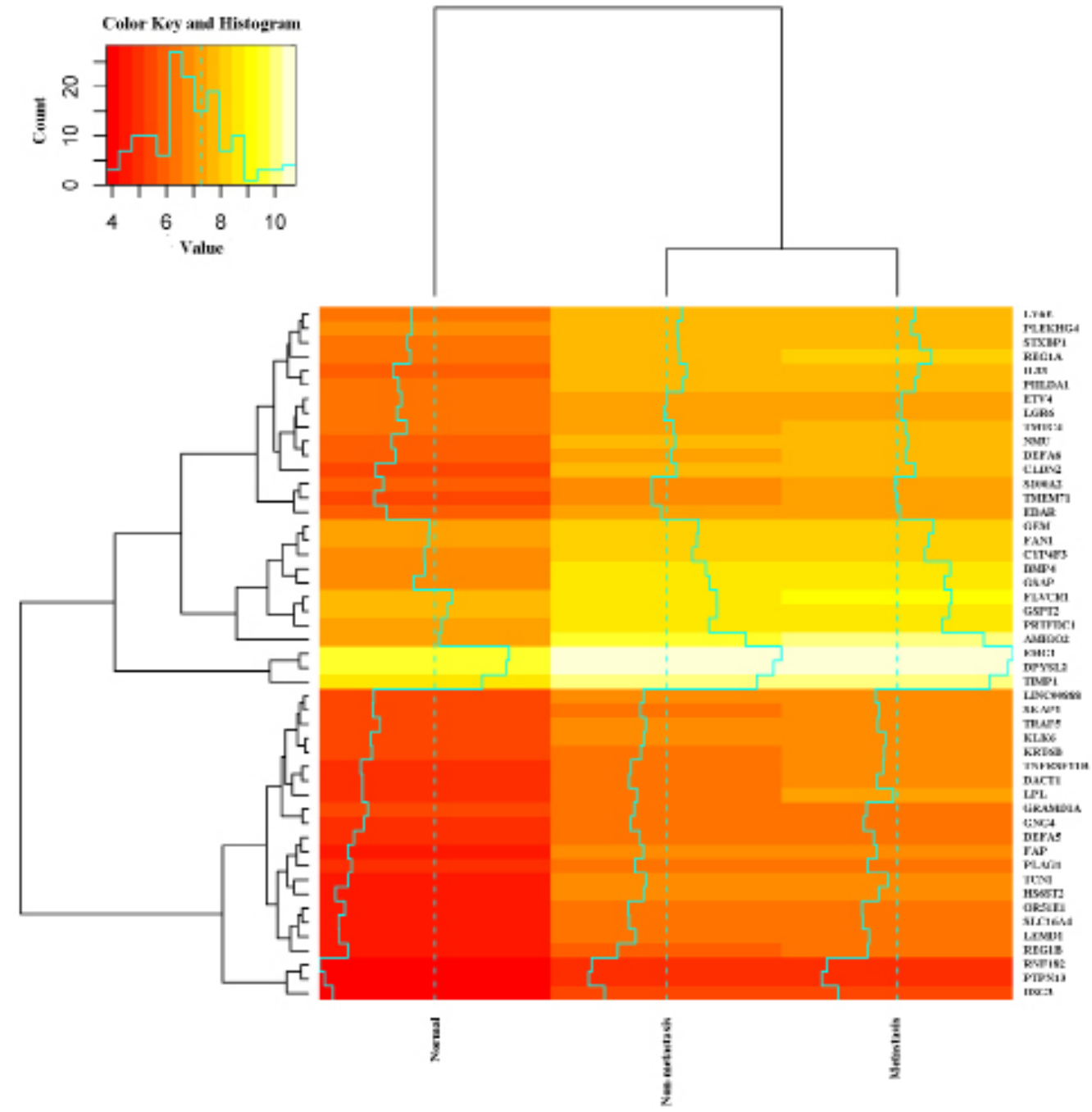

Figure 2. Heatmap of the average expression level of 49 critical genes in CRC samples with/without distant metastasis and normal samples. Metastasis indicates CRC samples with distant metastasis, non-metastasis indicates CRC samples without distant metastasis, and normal indicates normal samples. 


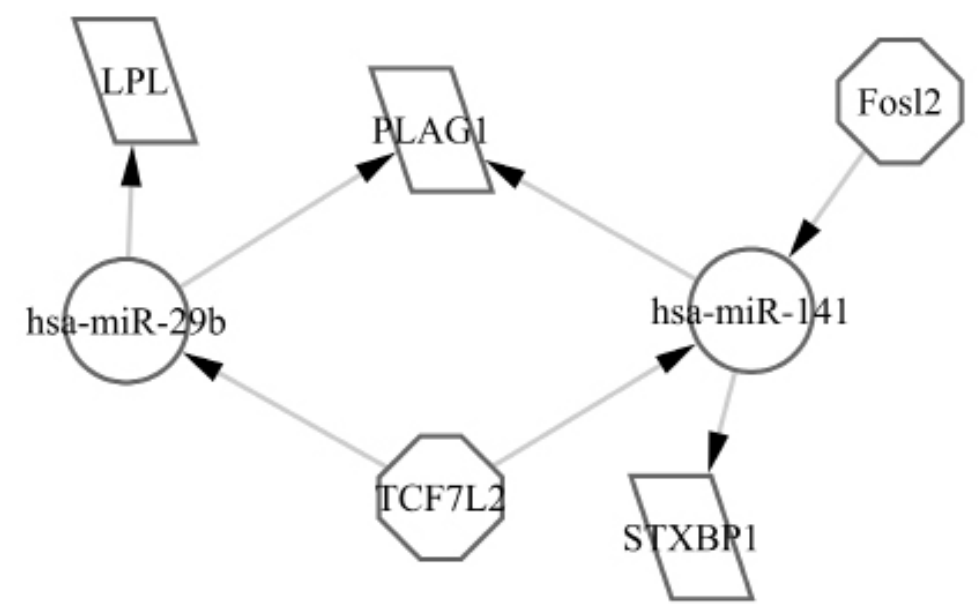

Figure 3. Regulation network composed by the six TF-miRNA-mRNA regulation loops. The octagon nodes represent TFs, circular nodes represent miRNAs, parallelogram nodes represent mRNAs, and arrows represent regulatory relationships.

\section{DISCUSSION}

Cancer metastasis is a complex process and many studies have been designed to explore cancer metastasis mechanisms and signatures. In this study, through the analysis of mRNA and miRNA expression profiles from the GEO database, DEGs and DE miRNAs that may be related to CRC metastasis were identified. Functional enrichment analysis of DEGs that are upregulated in CRC samples with distant metastasis than those in CRC samples without distant metastasis and normal samples indicated that these genes are mainly involved in cell differentiation or proliferation. Six critical TF-miRNA-mRNA regulation loops that may play important roles in CRC metastasis were identified.

A total of 49 DEGs were found to be upregulated in CRC samples with distant metastasis than that in CRC samples without distant metastasis and normal samples, these genes are thought to play important roles in CRC metastasis. Biological processes, related to cell proliferation and differentiation, were significantly enriched, which is consistent with previous studies (Arndt et al., 2009, Karagiannis et al., 2013). BMP4 was found in most of the GO terms. Many studies have clarified the relationship between BMP4 and CRC initiation or development. In an association study, Yang et al. (2014) found that rs4444235, a cis-acting regulator of $B M P 4$, could contribute to CRC risk in Taiwanese patients. The relationship between rs4444235 and CRC was also demonstrated by Lubbe et al. (2012). However, the association between BMP4 and CRC metastasis remains unclear and further studies are still needed.

Based on the starBase and ChIPBase databases, six TF-miRNA-mRNA regulation loops that may play important roles in CRC metastasis were identified. They include two TFs, two miRNAs, and three mRNAs. In the regulation loops, TCF7L2 regulates both miRNAs. As a transcript factor, $T C F 7 L 2$ plays a key role in the Wnt signaling pathway, which has been demonstrated to be involved in CRC (Ashktorab et al., 2014, Hu et al., 2014, Planutis et al., 2014). Additionally, the relationship between TF7L2 and CRC metastasis has been revealed 
in previous studies. Through whole genome sequencing, Shanmugam et al. (2014) detected a TCF7L2 mutation in metastatic CRC. In a large scale genetic study, Zhang et al. (2014) found that some loci in TCF7L2 and TGFB1 were associated with colorectal tumorigenesis.

hsa-miR-141 regulates two of the three mRNAs in the regulation loops. As a short non-coding RNA, hsa-miR-141 is involved in the post-transcriptional regulation of many target genes and its deregulation is associated with many types of cancer such as breast (Gregory et al., 2008) and kidney cancer (Nakada et al., 2008) as well as CRC and CRC metastasis. Through the investigation of some miRNA expression profiles, Yin et al. (2014) found that hsa-miR-141 was a novel biomarker in CRC liver metastasis. Cheng et al. (2011) identified hsa-miR-141 as a critical biomarker for metastatic CRC that predicts poor prognosis.

PLAG1 was regulated by both miRNAs, although no previous study proved the relationship between PLAG1 and CRC. However, it has been reported to be involved in other cancers. Dotlic et al. (2014) found that PLAG1 was involved in the development of neoplasm by using fluorescence in situ hybridization, reverse transcription-polymerase chain reaction, and gene microarray. Wang et al. (2013) illustrated the role of PLAG1 in pleomorphic adenoma through microarray analysis. Thus, PLAG1 may be a novel signature involved in CRC. In conclusion, through bioinformatics methods, critical targets that may be involved in CRC metastasis were identified. Some of these targets have been previously identified, while others are newly found targets, Wet laboratory experiments are warranted to confirm these newly found targets.

\section{CONCLUSIONS}

Overall, bioinformatics analysis of mRNA and miRNA expression profiles identified some TF-miRNA-mRNA regulation loops that may play important roles in CRC metastasis. These results will be helpful in experimental studies and clinical treatment of CRC.

\section{Conflicts of interest}

The authors declare no conflict of interest.

\section{ACKNOWLEDGMENTS}

Research supported by the National Natural Science Foundation of China (\#81101546).

\section{REFERENCES}

Arndt GM, Dossey L, Cullen LM, Lai A, et al. (2009). Characterization of global microRNA expression reveals oncogenic potential of miR-145 in metastatic colorectal cancer. BMC Cancer 9: 374-391.

Ashktorab H, Daremipouran M, Devaney J, Varma S, et al. (2014). Identification of novel mutations by exome sequencing in African American colorectal cancer patients. Cancer 121: 34-42.

Barrett T, Troup DB, Wilhite SE, Ledoux P, et al. (2011). NCBI GEO: archive for functional genomics data sets - 10 years on. Nucleic Acids Res. 39 (Database issue): D1005-1010.

Cheng H, Zhang L, Cogdell DE, Zheng H, et al. (2011). Circulating plasma MiR-141 is a novel biomarker for metastatic colon cancer and predicts poor prognosis. PLoS One 6: e17745.

Clifford RJ, Zhang J, Meerzaman DM, Lyu MS, et al. (2010). Genetic variations at loci involved in the immune response are risk factors for hepatocellular carcinoma. Hepatology 52: 2034-2043.

Coghlin C and Murray GI (2015). Biomarkers of colorectal cancer: recent advances and future challenges. Proteomics 
Clin. Appl. 9: 64-71.

Debouck C and Goodfellow PN (1999). DNA microarrays in drug discovery and development. Nat. Genet. 21: 48-50.

Diboun I, Wernisch L, Orengo CA and Koltzenburg M (2006). Microarray analysis after RNA amplification can detect pronounced differences in gene expression using limma. BMC Genomics 7: 252.

Dotlic S, Gatalica Z, Wen W, Ghazalpour A, et al. (2014). Extraskeletal myxoid chondrosarcoma of the vulva with PLAG1 gene activation: molecular genetic characterization of 2 cases. Appl. Immunohistochem. Mol. Morphol. 22: 537-542.

Gaiteri C, Ding Y, French B, Tseng GC, et al. (2014). Beyond modules and hubs: the potential of gene coexpression networks for investigating molecular mechanisms of complex brain disorders. Genes Brain Behav. 13: 13-24.

Gregory PA, Bert AG, Paterson EL, Barry SC, et al. (2008). The miR-200 family and miR-205 regulate epithelial to mesenchymal transition by targeting ZEB1 and SIP1. Nat. Cell Biol. 10: 593-601.

Herszenyi L, Barabas L, Hritz I, Istvan G, et al. (2014). Impact of proteolytic enzymes in colorectal cancer development and progression. World J. Gastroenterol. 20: 13246-13257.

Hu TH, Yao Y, Yu S, Han LL, et al. (2014). SDF-1/CXCR4 promotes epithelial-mesenchymal transition and progression of colorectal cancer by activation of the Wnt/beta-catenin signaling pathway. Cancer Lett. 354: 417-426.

Hu Y and Tang H (2014). MicroRNAs Regulate the Epithelial to Mesenchymal Transition (EMT) in Cancer Progression. Microrna 3: 108-117.

Huber W, Carey VJ, Gentleman R, Anders S, et al. (2015). Orchestrating high-throughput genomic analysis with Bioconductor. Nature Methods. 12: 115-121.

Joshi P, Middleton J, Jeon YJ and Garofalo M (2014). MicroRNAs in lung cancer. World J. Methodol. 4: 59-72.

Karagiannis GS, Berk A, Dimitromanolakis A and Diamandis EP (2013). Enrichment map profiling of the cancer invasion front suggests regulation of colorectal cancer progression by the bone morphogenetic protein antagonist, gremlin-1. Mol. Oncol. 7: 826-839.

Latchman DS (1993). Transcription factors: an overview. Int. J. Exp. Pathol. 74: 417-422.

Li JH, Liu S, Zhou H, Qu LH, et al. (2014). starBase v2.0: decoding miRNA-ceRNA, miRNA-ncRNA and protein-RNA interaction networks from large-scale CLIP-Seq data. Nucleic Acids Res. 42: D92-97.

Li L and Ma BB (2014). Colorectal cancer in Chinese patients: current and emerging treatment options. Onco Targets Ther. 7: 1817-1828.

Lubbe SJ, Pittman AM, Olver B, Lloyd A, et al. (2012). The 14q22.2 colorectal cancer variant rs 4444235 shows cis-acting regulation of BMP4. Oncogene 31: 3777-3784.

Lupinacci RM, Paye F, Coelho FF, Kruger JA, et al. (2014). Lymphatic drainage of the liver and its implications in the management of colorectal cancer liver metastases. Updates Surg. 66: 239-245.

Morris-Stiff G, White AD, Gomez D, Cameron IC, et al. (2014). Nodular regenerative hyperplasia (NRH) complicating oxaliplatin chemotherapy in patients undergoing resection of colorectal liver metastases. Eur. J. Surg. Oncol. 40: 1016-1020.

Nakada C, Matsuura K, Tsukamoto Y, Tanigawa M, et al. (2008). Genome-wide microRNA expression profiling in renal cell carcinoma: significant down-regulation of miR-141 and miR-200c. J. Pathol. 216: 418-427.

Ochiai T, Nishimura K, Watanabe T, Kitajima M, et al. (2014). Mechanism underlying the transient increase of serum iron during FOLFOX/FOLFIRI therapy. Mol. Clin. Oncol. 2: 968-972.

Planutis K, Planutiene M and Holcombe RF (2014). A novel signaling pathway regulates colon cancer angiogenesis through Norrin. Sci. Rep. 4: 5630

Shanmugam V, Ramanathan RK, Lavender NA, Sinari S, et al. (2014). Whole genome sequencing reveals potential targets for therapy in patients with refractory KRAS mutated metastatic colorectal cancer. BMC Med. Genomics 7: 36.

Shiah SG, Hsiao JR, Chang WM, Chen YW, et al. (2014). Downregulated miR-329 and miR-410 promote the proliferation and invasion of oral squamous cell carcinoma by targeting Wnt-7b. Cancer Res.74: 7560-7572.

Tsukamoto S, Ishikawa T, Iida S, Ishiguro M, et al. (2011). Clinical significance of osteoprotegerin expression in human colorectal cancer. Clin. Cancer Res. 17: 2444-2450.

Vali L, Hahn O, Kupcsulik P, Drahos A, et al. (2008). Oxidative stress with altered element content and decreased ATP level of erythrocytes in hepatocellular carcinoma and colorectal liver metastases. Eur. J. Gastroenterol. Hepatol. 20: 393-398.

Wang Y, Shang W, Lei X, Shen S, et al. (2013). Opposing functions of PLAG1 in pleomorphic adenoma: a microarray analysis of PLAG1 transgenic mice. Biotechnol. Lett. 35: 1377-1385.

Xu Y, Xu Q, Yang L, Ye X, et al. (2013). Identification and validation of a blood-based 18-gene expression signature in colorectal cancer. Clin. Cancer Res. 19: 3039-3049.

Yang CY, Lu RH, Lin CH, Jen CH, et al. (2014). Single nucleotide polymorphisms associated with colorectal cancer susceptibility and loss of heterozygosity in a Taiwanese population. PLoS One 9: e100060.

Yang JH, Li JH, Jiang S, Zhou H, et al. (2013). ChIPBase: a database for decoding the transcriptional regulation of long non-coding RNA and microRNA genes from ChIP-Seq data. Nucleic Acids Res. 41: D177-187. 
Yesudhas D, Gosu V, Anwar MA and Choi S (2014). Multiple roles of toll-like receptor 4 in colorectal cancer. Front Immunol. 5: 334 .

Yin J, Bai Z, Song J, Yang Y, et al. (2014). Differential expression of serum miR-126, miR-141 and miR-21 as novel biomarkers for early detection of liver metastasis in colorectal cancer. Chin. J. Cancer Res. 26: 95-103.

Zhang B, Jia WH, Matsuda K, Kweon SS, et al. (2014). Large-scale genetic study in East Asians identifies six new loci associated with colorectal cancer risk. Nat. Genet. 46: 533-542.

Zhang Y and Wang XF (2014). Post-transcriptional regulation of MTA family by microRNAs in the context of cancer. Cancer Metastasis Rev. 33: 1011-1016.

Zhou JJ, Zheng S, Sun LF and Zheng L (2014). MicroRNA regulation network in colorectal cancer metastasis. World J. Biol. Chem. 5: 301-307.

Zhu Z, Zhang X, Wang G and Zheng H (2014). Role of MicroRNAs in Hepatocellular Carcinoma. Hepat. Mon. 14: e18672. 